\title{
STUDY OF ANTENNAS IN C BAND FOR SATELLITE COMMUNICATION
}

\author{
Hiteshwer Rohilla*1, Monish Batra ${ }^{2}$, Krishan Kumar \\ *1,2,3 dronacharya College Of Engineering
}

*Corresponding Author: -

\begin{abstract}
: -
The main focus of this paper on the various types of antennae used in $C$ band for satellite communication. This paper gives information to reader about the antennas used for $C$ band. It gives overview about the patch antenna and dish antenna and their individual losses and performance. In the end of paper, we discuss some of antennas that can be used for future aspects in satellite communication.
\end{abstract}

Keywords: $C$ band, Antenna, microstrip patch 


\section{INTRODUCTION:}

$\mathrm{C}$ band for satellite communication is electromagnetic frequency band. $\mathrm{C}$ band is divided into two standards. One is IEEE C band and another one is NATOC band. IEEECbandincludes frequency rangefrom 4 to $8 \mathrm{GHzandNATOCbandincludes} \mathrm{frequency} \mathrm{range}$ 500 to $1000 \mathrm{MHz}$. Cband is less affected by adverse weather conditionlikerainattenuationand skynoiseascomparetoKuband. Cbandare used for applications like weather radar system, voice and other data communication applications. There are various types of antennas used in $\mathrm{C}$ band forsatellitecommunicationsfordifferentapplicationbutmostlypatchantennais used.

\section{Microstrip Patch Antenna}

A microstrip patch antenna is very small antenna that have a conducting metal plate on one side of substrate and conducting ground plane on another side of substrate. It is constructed on a dielectric substrate using lithography process. Microstrip patch antenna is very small in size and installed in microstrip array form. Typically, these antennae have gain of 5 to $6 \mathrm{db}$. These shows a good performance and canbeinstalledinnanosatellites. Thereis various designof microstrip antennas like rectangular microstrip antenna, circular microstrip and in elliptical shape antenna, each have their own characteristics.

\subsection{GAIN}

Gain of antenna is represents the radiation efficiency. It is defined as the ratio of radiationproducedbyafar-fieldtotheradiation producedbyanisotropicsource. Gainof this antennaissmalltypically5to6db.Gaincanbeincreasedbyuseof array of microstrip patch antennas. The rectangular patch antenna has maximum directivity in one direction to the direction of broadside. Gain of this antenna reducedduetothesurfacewavelossesandlossesduetothedielectricmedium used.

\subsection{IMPEDANCE}

Input impedance of a patch antenna depends on quality factor, resonant frequency and the resonant resistance.

$$
F_{C} \approx c / 2 L \sqrt{ } \varepsilon r
$$

As the spacing between the patch and the ground plane decreases lesser energy is released and more energy is stored in patch capacitance and inductance

The width of microsrtip antennas controls the input impedance. Larger the width larger will bethebandwidth. For square patch antennas theinputimpedance will be on the order of $300 \mathrm{Ohms}$. The impedance can be reduced by increasing the width, so to decreasetheinputimpedanceto50 Ohms widepatchantennasare required, which takes up a lot of valuable space.

\subsection{ADVANTAGES:}

An array of patch antenna is used rather than a single antenna which provides more gain than a single patch antenna. It can be easily manufacture as it includes two-dimensional geometry. It supports both linear and circular polarization. These are efficient radiators. Due to smallsize these haveless weight. Althoughthese havelowmanufacturing cost.Someofdisadvantagesof patch antennaare:

Excitation of surfacewave.

Low impedance bandwidth.

Extra radiation occurs from its feeds and junctions.

These antennas can be directly printed on the PCB.

\section{Satellites Dish Antenna}

Asatellitedishantennaisaparabolic shapeofdishthatreflectssignalstodish's focalpoint.Ithasafeedhornthatismountedondish thatisbasicallyawaveguide that couple electromagnetic energy into the cable. This feedhorn gathers the signal to aLNB.LNB is a lownoise block downconverter device that is a type of low noise amplifier. The distance between the feed antenna and the reflector is typically several wavelengths as well. This is in contrast to the corner reflector, where the antenna is roughly a halfwavelength from the reflector.

\subsection{GAIN}

Parabolic reflector orlarge dish have largergain commonly from 30 to $40 \mathrm{db}$ and also have low cross polarization. They have reasonable bandwidth approx. 5\%. Unlike resonant antennas like the dipole antenna which are typically approximately a half-wavelength long at the frequency of operation, the reflectingdishmustbemuchlargerthanawavelengthinsize. Thedish isatleast several wavelengths in diameter, but the diameter can be on the order of 100 wavelengths for very high gain dishes (>50 dB gain).

\subsection{ADVANTAGES}

The Parabolic antennas has high directivity. Parabolic antennas possess some of top gains, that is they can develop narrowest bandwidths, connected with almost any feeler type. Parabolic antennas are used in high frequency part of radio spectrum, at uhf and microwave frequencies. It can used as a high gain antenna forapplicationslikemicrowavelinksthatcarrytelephoneand televisionsignals.

\section{Future antennas:}

With the advancement in technology and the trend of ever increasing capcity availability of services leads to more flexible , lighter and even more stylish satellite antennas. Combining Tx andRxintosameantennaisafeature of new satelliteantennas. Theimplementation of PIM-freemultibandantennaswillcall for advanced low-loss multiplexer technologies such as the 
triplexers and quadruplexers that have been required on some recent programs.

Reconfigurable antennas: In FSS/DSB communication need of reconfigurable antennas is increased in last few years. Reconfigurable antennahave the ability to reconfigure their shape by mechanical means or by electronic means. They provide flexibility to operator to change the shape of spacecraft in orbit in order to cope with change in traffic requiremnets.

Active Direct Radiating Array (DRA) is another future antenna that offersthe potential for unequaled coverage flexibility from space, with significant commercial returns

\section{Conclusion:}

Inthisarticleweconcluded satellitedishandmicrostripantennasthatisusedin satellitec bandcommunicationand theirdifferent paramenters. Dish antennas have larger in dimension but they have larger gain than microstrip patch antenna. While microstrip patch antenna have small size and used in many application where small size antennas required like in nanosatellite and in small size satellites.

\section{References:}

[1].http://www.antenna-theory.com

[2]. Microstrippatchantennaandits application:Asurveyby IndersenSingh, Dr. VS triphthi

[3]. http://www.satmagazine.com 